\title{
Pattern of Smoking Among Tuberculosis Patients : An Analysis in A Tertiary Care Hospital
}

\author{
Ahmed Tanjimul Islam ${ }^{*}$ \\ Md Jawadul Haque ${ }^{2}$ \\ Md Azizul Hoque \\ Rubab Tarannum Islam ${ }^{3}$
}

'Department of Medicine Rajshahi Medical College Hospital Rajshahi, Bangladesh

${ }^{2}$ Department of Community Medicine Rajshahi Medical College Hospital Rajshahi, Bangladesh

'Department of Medicine Rajshahi Medical College Hospital Rajshahi, Bangladesh.

${ }^{3}$ Department of Pharmacy University of Science \& Technology Chittagong (USTC) Chittagong, Bangladesh.

\section{*Correspondence to:}

\section{Dr. Ahmed Tanjimul Islam}

Medical Officer of Medicine

Rajshahi Medical College Hospital

Rajshahi, Bangladesh.

Email: droveesomch@gmail.com

Mobile: +8801554333026

\begin{abstract}
Background: Tuberculosis is among the major causes of illness and death worldwide especially in Asia. Smoking is associated with recurrent tuberculosis and its related mortality. Also, it could affect clinical manifestations, bacteriological conversion and outcome of treatment. This study aimed to evaluate the pattern of tobacco smoking, history of previous quit attempts and attitude towards quitting in tuberculosis patients. Materials and Methods: It was a cross-sectional study done amongst tuberculosis patients presented to DOTS corner of Rajshahi Medical College Hospital. 315 patients entered the study as "First Come First Serve". Selfreported questionnaires were designed according to the standard questionnaires of smoking pattern. Results: Sixty one percent $(61 \%)$ patients $(n=192)$ were smoker before the diagnosis of tuberculosis. $51.1 \%$ were current smokers at the time of interview and were continuing smoking after the diagnosis of tuberculosis. Only $10.2 \%$ discontinued smoking after diagnosis. $68.2 \%$ smokes less than 10 cigarettes per day. $53.1 \%$ was between $31-50$ age group. $21-30$ is the commonest age group $(62 \%)$ to start smoking is and $66.1 \%$ smoked less than 10 years before diagnosis. To reduce stress was the most common cause to continue smoking (48.9\%). $71.9 \%$ smokers showed keen interest to stop smoking. Conclusion: Considering the prevalence of smoking in tuberculosis patients, evaluation of tobacco smoking status in such patients and motivating them to quit smoking could be considered as important steps in their treatment process.
\end{abstract}

Key words: Tuberculosis; Smoking pattern; Tobacco.

\section{INTRODUCTION}

Chronic diseases are conditions that require years or even decades of constant care and are usually associated with irreversible manifestations and an apparent or hidden constellation of disease complications or injuries. In other words, chronic conditions result in gradual but stable physical and mental deterioration affecting patients' quality of life. These conditions result in decreased function, and impaired personal and social relations ${ }^{1}$. Tuberculosis is amongst the most common causes of morbidity and mortality in the world especially in Asia. In 2006, 9.2 million new tuberculosis cases and 1.7 million deaths due to tuberculosis were reported globally ${ }^{1,2}$. On the other hand, cigarette smoking is amongst the most preventable causes of mortality and the second cause of death worldwide.

At present, smoking is the cause of one out of every 10 deaths that occur. According to the WHO estimates, the rate of morbidity and mortality due to tobacco consumption will reach 8 million by the year $2030^{2}$. However, the growing trend of tobacco consumption among adolescents and the youth has been worrisome. 
A correlation between tobacco consumption and tuberculosis was suggested and confirmed by multiple studies and has become the center of attention during the recent years ${ }^{3,4}$. Current studies have highlighted several correlations between cigarette smoking and tuberculosis infection ${ }^{4}$. Tobacco consumption is directly correlated with recurrence and disability due to tuberculosis. Also, cigarette smoking impacts clinical manifestations, bacteriologic conversion and treatment outcome of disease ${ }^{5}$. In other words, one of every 5 deaths due to tuberculosis could have been prevented if the patient was not a smoker ${ }^{6}$. That is why tuberculosis patients should be a target of smoking cessation programs. Disease recurrence is one of the most important challenges encountered during the course of treatment in tuberculosis patients. Treatment compliance is another important factor for successful treatment of tuberculosis. Absence from work in smoker tuberculosis patients may be due to their nicotine addiction. So, By Collecting information regarding the pattern of tobacco consumption and prevalence of smoking, our knowledge can be enhanced in order to prevent and control cigarette smoking.

\section{MATERIALS AND METHOD}

Study design and data collection

This was a cross-sectional study conducted during eight month period from July 2014 to February 2015. All patients presented to the DOTS Corner of Rajshahi Medical College Hospital during this time period entered the study. Data were collected by trained technicians through face to face interview with patients. Only patients who were fully alert and conscious entered the study after obtaining a written informed consent from them. The questionnaire contained 23 questions and was designed according to the IUATLD questionnaire for tobacco consumption and ASI (Addiction Severity Index) questionnaire for smoking and substance abuse. Data regarding age of initiation of smoking, pattern of smoking, the first experience of cigarette offering, quit attempts and number of cigarettes they smoked per day was collected and entered the questionnaires.

\section{Statistical analysis}

Survey data were analyzed using the Statistical Package for Social Sciences (SPSS Inc, Chicago, IL, USA). The numbers of cigarettes smoked were numerically added together to obtain an aggregate score of the quantity smoked daily. Descriptive statistical analyses were performed and a $p$ value $<0.05$ was considered statistically significant.

Ethical considerations

Institutional Review Board (IRB) approval was obtained from ethical committee of Rajshahi Medical College and Hospital. Before administering the survey, investigators explained the purpose of the study to all patients. The voluntary nature of participation and the anonymous and confidential nature of the interview schedules were strongly emphasized. Verbal informed consent was obtained from all patients.

\section{RESULTS}

Among 315 tuberculosis patients who were selected for this study, 192 patients $(61 \%)$ found as smoker before diagnosis. Despite of diagnosis of tuberculosis almost half of the patients (51.8\%) were continuing smoking. Only a few numbers of patients (10.2\%) discontinued smoking after diagnosis of tuberculosis. 123 tuberculosis patients were found non smoker (Table 1).

Table1: Current smokers among tuberculosis patients $(\mathrm{n}=315)$.

$\begin{array}{lcc}\text { Current smoking status } & \text { Number } & \text { Percentage } \\ \text { Continuing smoking } & 160 & 50.8 \% \\ \text { Discontinuing smoking } & 32 & 10.2 \% \\ \text { Non smoker } & 123 & 39 \% \\ \text { Total } & 315 & 100 \%\end{array}$

Among the 192 tuberculosis smokers, 68.2\% (n=131) smoked less than 10 cigarettes per day. Other $21.4 \%$ smoked more than 10 cigarettes with $10.4 \%(n=20)$ smoked more than 20 cigarettes per day (Table 2).

Table 2: Distribution of smokers based on number of cigarettes smoked per day.

\begin{tabular}{lrc} 
Number of Cigarettes & Number of Patients & Percentage \\
$<10$ & 131 & $68.2 \%$ \\
$10-20$ & 41 & $21.4 \%$ \\
$>20$ & 20 & $10.4 \%$ \\
Total & 192 & $100 \%$ \\
\hline
\end{tabular}

We found that, younger age group are the most vulnerable for smoking in tuberculosis. More than half $(53.1 \%)$ were in the age group 31-50 years. Older age people ( $>50$ years) had less smoking (10.9\%) compared to younger age group (Table 3).

Table 3 : Age wise distribution of number of smoker in Tuberculosis.

\begin{tabular}{lrc} 
Age group & Number & Percentage \\
$<30$ & 69 & $36 \%$ \\
$31-50$ & 102 & $53.1 \%$ \\
$>50$ & 21 & $10.9 \%$ \\
Total & 192 & $100 \%$ \\
\hline
\end{tabular}

Younger age groups were also more prone to start smoking habit. 21-30 were the commonest age group to start smoking in tuberculosis patients. $28.1 \%$ started smoking before the age of 20 . Only 7 tuberculosis started smoking after the age 40 (Table 4).

Table 4 : Distribution of smokers based on initiation age of smoking.

\begin{tabular}{lrc} 
Starting Age Group & Number & Percentage \\
$<20$ & 54 & $28.1 \%$ \\
$21-30$ & 119 & $62 \%$ \\
$31-40$ & 12 & $6.3 \%$ \\
$>40$ & 7 & $3.6 \%$ \\
Total & 192 & $100 \%$ \\
\hline
\end{tabular}

Our study also revealed that most of the patients smoked for a long period of time before diagnosis of tuberculosis. $66.1 \%$ smoked for at least 10 years. 59 patients smoked around 10-20 years. Only 6 patients smoked for more than 20 years (Table 5). 
Table 5 : Distribution of smokers based on number of years smoked.

\begin{tabular}{lcc} 
Years smoked & Number & Percentage \\
$<10$ & 127 & $66.1 \%$ \\
$10-20$ & 59 & $30.8 \%$ \\
$>20$ & 6 & $3.1 \%$ \\
Total & 192 & $100 \%$ \\
\hline
\end{tabular}

This study also explored the reasons of continuing smoking after the diagnosis of tuberculosis. Patients said multiple reasons for the smoking. To reduce stress $(48.9 \%)$ were the commonest cause to continue smoking. 78 patients smoked only to get pleasure. People's influence, self confidence, enhance work performance were the other common causes (Table 6).

Table 6 : Reason to continuing Smoking after tuberculosis diagnosis.

\begin{tabular}{lcc} 
Reasons & Number & Percentage \\
Reduce stress & 94 & $48.9 \%$ \\
To get pleasure & 78 & $40.6 \%$ \\
Peer pressure & 54 & $28.1 \%$ \\
Self confidence & 33 & $17.2 \%$ \\
Enhance work performance & 31 & $16.1 \%$ \\
\hline
\end{tabular}

*Allowed to answer multiple reasons.

Among the smokers of tuberculosis, a good number of patients $(71.9 \%)$ showed interest to quit smoking after diagnosis of Tuberculosis. 33 patients showed confidence to quit smoking in recent days. Only few patients $(10.9 \%)$ showed no confidence to quit smoking (Table 7).

Table 7: Smoker's future plan to quit smoking.

\begin{tabular}{lcc} 
Future plan to quit & Number & Percentage \\
Interest to quit & 138 & $71.9 \%$ \\
Will attempt to quit & 33 & $17.2 \%$ \\
Not confident & 21 & $10.9 \%$ \\
Total & 192 & $100 \%$ \\
\hline
\end{tabular}

\section{DISCUSSION}

Our study results revealed the fact that many of tuberculosis patients are active or occasional smokers. In a study conducted by Gullón Blanco and colleagues on the impact of smoking on sputum smear of tuberculosis patients, $64 \%$ of patients were smokers ${ }^{5}$. Considering our study findings, smoking cessation interventions by the medical staff implementing the DOTS seem necessary. In our study, more than half of smoker tuberculosis patients had previous quit attempts and most of them had done it personally with no help from outside. In a study conducted in Indonesia it was shown that $72 \%$ of tuberculosis patients had tobacco consumption ${ }^{6}$. By using smoking cessation interventions, this rate decreased to $11 \%$ at the time of next visit.
This figure reduced further to $2 \%, 1-3$ months after treatment. In the same study, amongst 139 cases that were evaluated, only $30 \%$ were asked about the habit of smoking.

As mentioned earlier, in our study only $0.5 \%$ of cases had used medications in order to quit smoking which justifies the need for comprehensive and fundamental smoking cessation programs. Also, the knowledge of tuberculosis patients about the health hazards of smoking was relatively high in our study which was in accord with the previous study findings ${ }^{7}$. In their study, $94 \%$ of smoker tuberculosis patients who quit smoking and $56 \%$ of those who did not succeed to quit believed that even smoking one cigarette is hazardous to the health. It seems that high knowledge of our study patients about the adverse effects of smoking on the lungs may be due to the fact that these patients had a pulmonary disease and experienced related complications. Smoking cessation consultation for smoker TB patients should focus on enhancing their knowledge about the hazardous effects of smoking on health and especially their disease $^{8}$. Considering the prevalence of tobacco consumption among tuberculosis patients, evaluation of the cigarette smoking status and encouraging them to quit play an important role in control and treatment of tuberculosis ${ }^{8}$. Adding behavioral studies and nicotine replacement therapies to the DOTS can result in faster recovery, shorter infectious period and prevent treatment failure 9 . Prevention of tobacco consumption and encouraging people to stop smoking can decrease the incidence of clinical cases of tuberculosis and related deaths ${ }^{10}$. Prevalence of tobacco consumption in TB patients was greater than similar healthy subjects in our country. According to Thomas et al. study, tobacco consumption is an independent predictive factor in $\mathrm{TB}$ recurrence (OR: 3.1\%, 95\% Confidence Interval (CI): 1.6-6.0).

\section{CONCLUSION}

It looks like tuberculosis and tobacco consumption epidemics will continue their growing trend and developing countries will still be involved-even more than before with the adverse effects and consequences of these two major public health hazards. In this respect, merging the two systems suggested by the WHO namely DOTS for tuberculosis and MPOWER for tobacco control and enhancing each one by the other can be a great strategy for controlling these obstacles. By promoting smoking cessation in TB patients, we can increase patients' compliance to treatment, improve their interpersonal and social communications, decrease their stress, and control their risky behaviors.

\section{DISCLOSURE}

All the authors declared no competing interest. 


\section{REFERENCES}

1. WHO report 2008, Global tuberculosis control surveillance, planning, financing. www.who.int/tb/publications/global_ report/2008/pdf/fullreport.pdf.

2. WHO report on the global tobacco epidemic, 2008. The MPOWER Package. http://www.who.int/tobacco/ mpower/en/index/html.

3. Mohammad K, Noorbala AA, Majdzadeh SR, Karimloo M. Trends of Prevalence of Tobacco consumption in Iran (1370- 78). According to two National Health and disease projects. Hakim Journal. 2000; 197: 290- 294.

4. Gullón Blanco JA, Suárez Toste I, Lecuona Fernández M, Galindo Morales R, Fernández Alvarez R, Rubinos Cuadrado G et al. Tobacco smoking and sputum smear conversion in pulmonary tuberculosis. Med Clin (Barc). 2007; 128 (15): 565- 568.

5. Slama K, Chiang CY, Enarson DA. Educational series: Tobacco and Tuberculosis. Tobacco cessation intervention for tuberculosis patients. Int J Tuberc Lung Dis. 2007; 11(6): 612- 616.

6. Abal AT, Jayakrishnan B, Parwer S, El Shamy A, Abahussain E, Sharma PN. Effect of cigarette smoking on sputum smear conversion in adults with active pulmonary tuberculosis. Respir Med. 2005; 99 (4): 415- 420.

7. Leung CC, Li T, Lam TH, Yew WW, Law WS, Tam CM et al. Smoking and tuberculosis among the elderly in Hong Kong. Am J Respir Crit Care Med. 2004; 170 (9): 1027- 1033.

8. Gajalakshmi V, Peto R, Kanaka TS, Jha P. Smoking and mortality from tuberculosis and other diseases in India: Retrospective study of 43000 adult male deaths and 35000 controls. Lancet. 2003; 362 (9383): 507- 515.

9. Thomas A, Gopi PG, Santha T, Chandrasekaran V, Subramani R, Selvakumar N et al. Predictors of relapse among pulmonary tuberculosis patients treated in a DOTS program in South India. Int J Tuberc Lung Dis. 2005; 9 (5): 556- 561.

10. El Sony A, Slama K, Salieh M, Elhaj H, Adam K, Hassan A et al. Feasibility of brief tobacco cessation advice for tuberculosis patients: A study from Sudan. Int J Tuberc Lung Dis. 2007; 11 (2): 150155. 